\title{
Air Injection as a Solution to the Problem for Reducing High-Frequency Acoustic Noise in Some Operating Modes of Francis Hydro Turbines
}

\author{
E. N. Dolmatov ${ }^{1 *}, S . Y$. Ilin $^{1}$ and $V . V$. Eliferov ${ }^{1}$ \\ ${ }^{1}$ JSC «Power Machines», Leningradsky Metallichesky Zavod (LMZ), Vatutina St., 3A, Saint- \\ Petersburg, Russia, 195009
}

\begin{abstract}
During operation of Francis hydro turbines at the regimes of 70 $95 \%$ of Nrated, high-frequency acoustic phenomena (noise) were recorded. In order to study and identify the causes of these phenomena, special field tests were conducted. The main objectives of the tests were: determination of the main frequencies of acoustic phenomena, comparison with natural frequencies of the turbine water passages elements and the search for solutions to reduce acoustic phenomena. During the tests, the natural frequencies of the elements of turbine water path were investigated. Several methods of air injection to the turbine water path were also tested. The most effective way (including by the amount of air) to reduce acoustic phenomena was the air injection from industrial air piping into the spiral case and into the area of guide vanes.
\end{abstract}

\section{Introduction}

At one on the HPP in Kazakhstan several years ago, two of the four units were renovated. The renovation was also included a runner replacement.

The main technical parameters of the original and new runner are shown in the table 1.

Table 1. The main technical parameters of the original and new runner

\begin{tabular}{|c|c|c|}
\hline Parameter & Original runner & New runner \\
\hline Runner diameter, $\mathrm{m}$ & 5.45 & 5.45 \\
\hline Number of runner blades & 14 & 13 \\
\hline Number of guide vanes & 24 & 24 \\
\hline Rated net head, $\mathrm{m}$ & 39.8 & 39.8 \\
\hline Maximum net head, $\mathrm{m}$ & 41.8 & 41.8 \\
\hline Rotation frequency, $\mathrm{min}^{-1}$ & 83.3 & 83.3 \\
\hline
\end{tabular}

* Corresponding author: dolmatov_en@ power-m.ru 


\begin{tabular}{|c|c|c|}
\hline Parameter & Original runner & New runner \\
\hline Maximum power at rated net head, MW & 85 & 95 \\
\hline
\end{tabular}

During operation of renovated units with new runner at the regimes of $70-95 \%$ of maximum power, high-frequency acoustic phenomena (noise) were fixed. Noise has dominant frequencies about 300-370 Hz. On units with old runners such phenomena were not observed. Decrease in the number of runner blades do not lead to the occurrence of new resonant frequencies close to $300-370 \mathrm{~Hz}$ (multiplication of rotation, vane and blade frequencies gives $433 \mathrm{~Hz}$ ).

In order to study and identify the causes of these phenomena, special field tests were conducted.

The main objectives of the tests were: determination of the main frequencies of acoustic phenomena, comparison with natural frequencies of the turbine water passages elements and the search for solutions to reduce acoustic phenomena.

\section{Determination of the main frequencies of acoustic phenomena}

During special tests sound pressure measurements in the turbine pit were made by microphone. Main spectrum of these measurements is presented on figure 1. At regimes 70$85 \%$ of maximum power dominant frequency of sound is about $300-310 \mathrm{~Hz}$, at regimes $85-$ $95 \%$ frequency of sound is $340-350 \mathrm{~Hz}$ (figure 2).

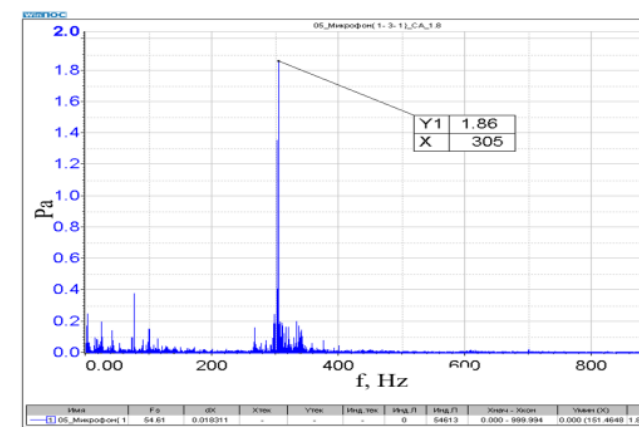

(a)

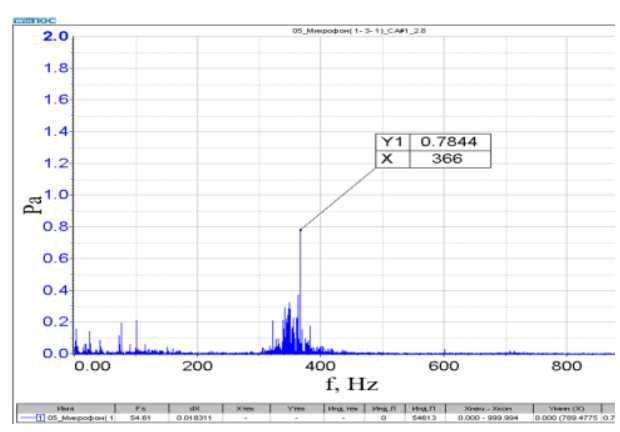

(b)

Fig. 1. Spectrum of sound pressure in turbine pit at $75 \mathrm{MW}$ (a), $85 \mathrm{MW}$ (b).

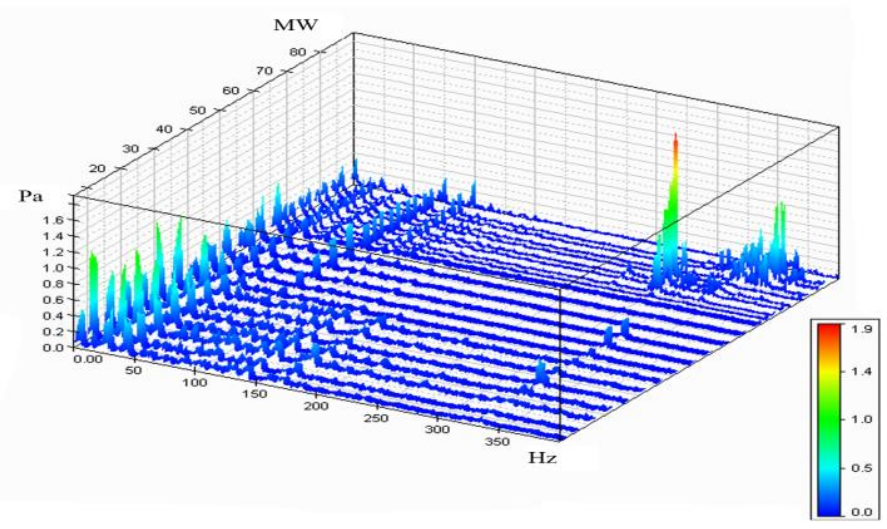

Fig. 2. 3D-spectrum of sound pressure in turbine pit. 


\section{Results of special vibration tests}

Vibrations of different parts of hydro unit were also measured in parallel with sound pressure measurements. Vibration sensor were installed on the generator spider, the head cover, turbine bearing, the regulating ring, stem of guide vane in axial direction, on the lever of guide vane in circumferential direction (figure 3).

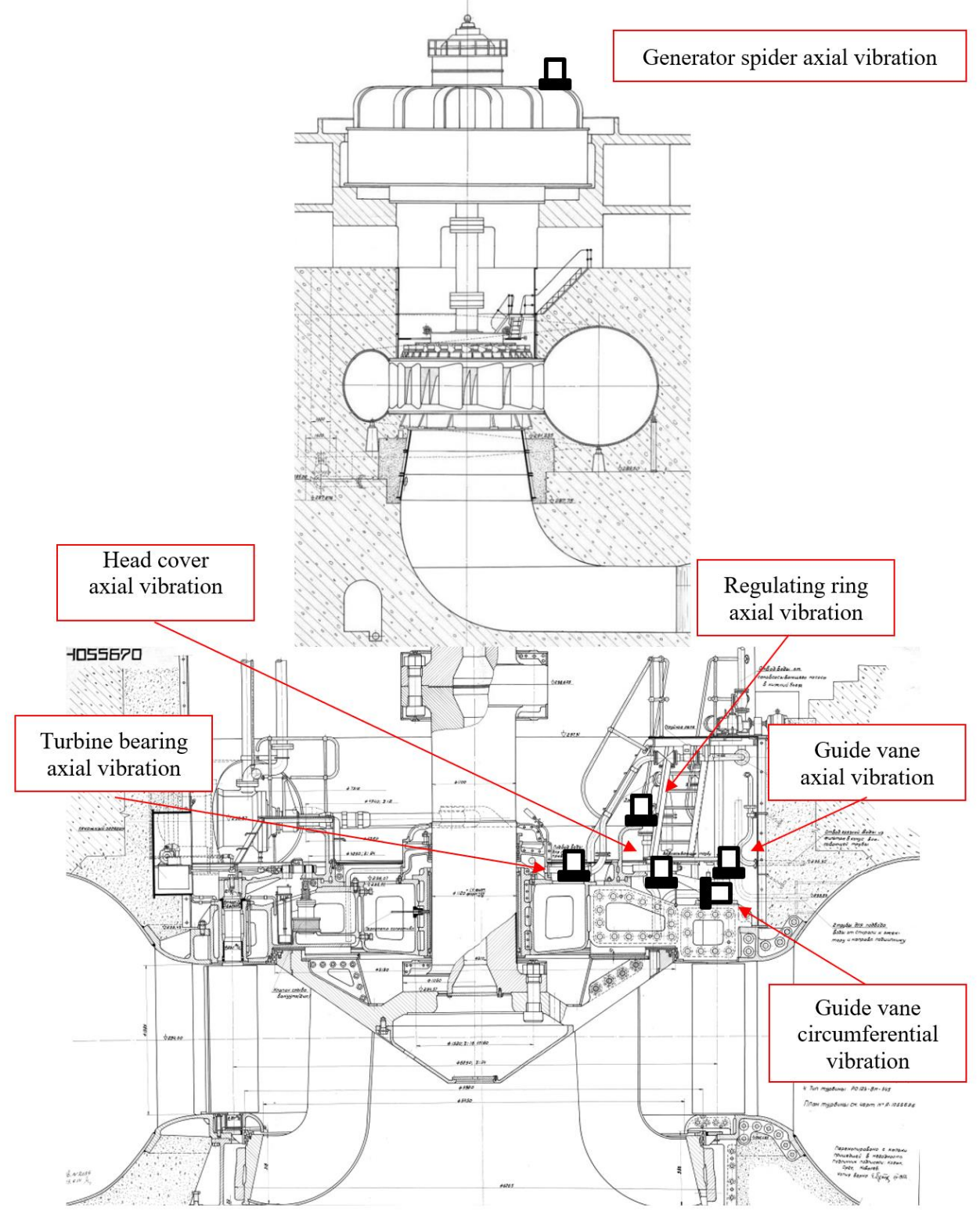

Fig. 3. Location of vibration sensors. 
It is shown on figure 4 that the highest vibrations at 70-90\% of maximum power (high noise zone) were measured on the regulating ring in axial direction and on the lever of guide vane in circumferential direction.

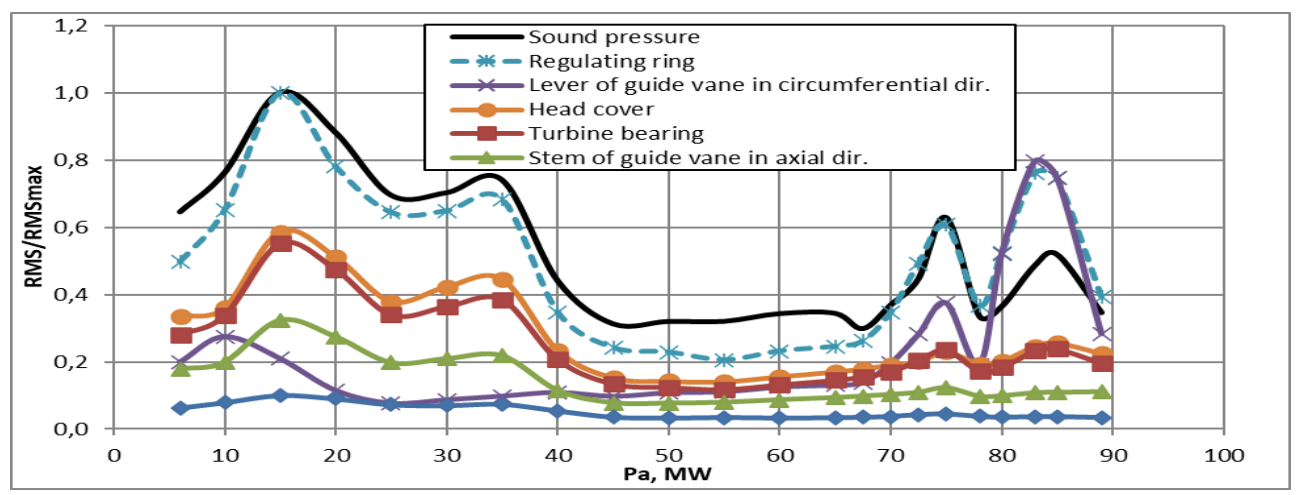

Fig. 4. Results of vibration and sound pressure measurements.

It was detected that regulating ring vibrations in axial direction (figure 5) and guide vane vibrations in circumferential direction (figure 6) have the same main frequencies 300-370 $\mathrm{Hz}$ as sound pressure (noise) at 70-90\% of maximum power (figure 2). This fact and that regulating ring is structurally connected to guide vanes may indicate that acoustic phenomena are connected with guide vane vibrations in circumferential direction.

The most likely source of guide vane vibration is a vortex forming after vane.

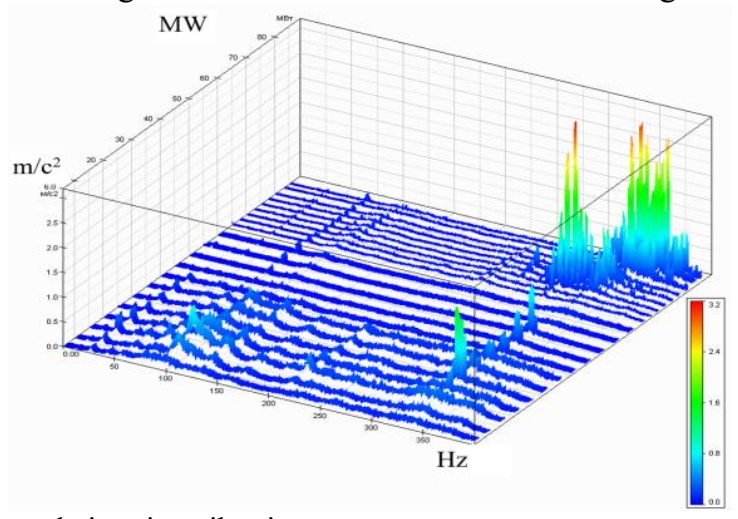

Fig. 5. 3D-spectrum of regulating ring vibration.

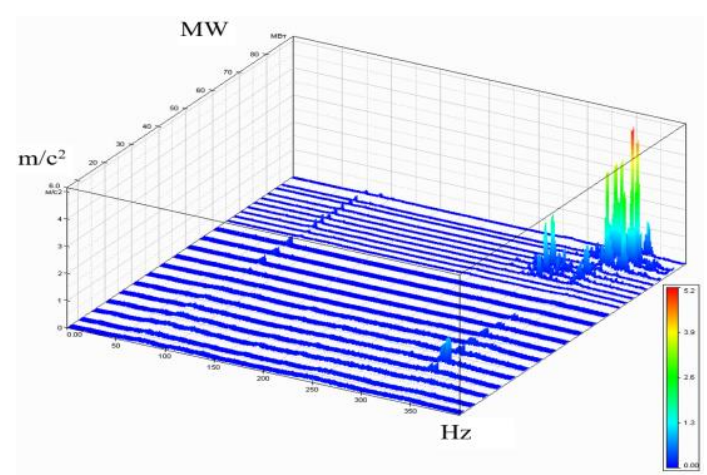

Fig. 6. 3D-spectrum of guide vane vibration in circumferential direction. 


\section{Determination of the natural frequencies of the elements of turbine}

During the tests, the natural frequencies of the elements of turbine water path were investigated. The goal was to find for natural frequencies of elements close to the fundamental frequency of the noise, that is, to determine an element that could be in a resonance and be a noise source. Impact method was used to determine the natural frequencies in the air. First natural frequencies of stay vane, guide vane and runner blade are shown at figures 7,8 and 9 respectively.

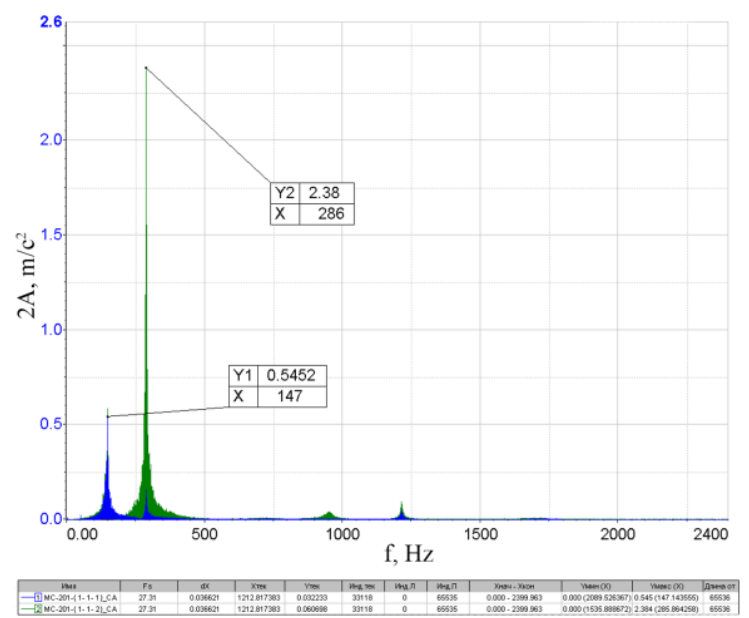

Fig. 7. First natural frequencies of stay vane (blue color - the sensor is centered; green color - the sensor is located at the edge).

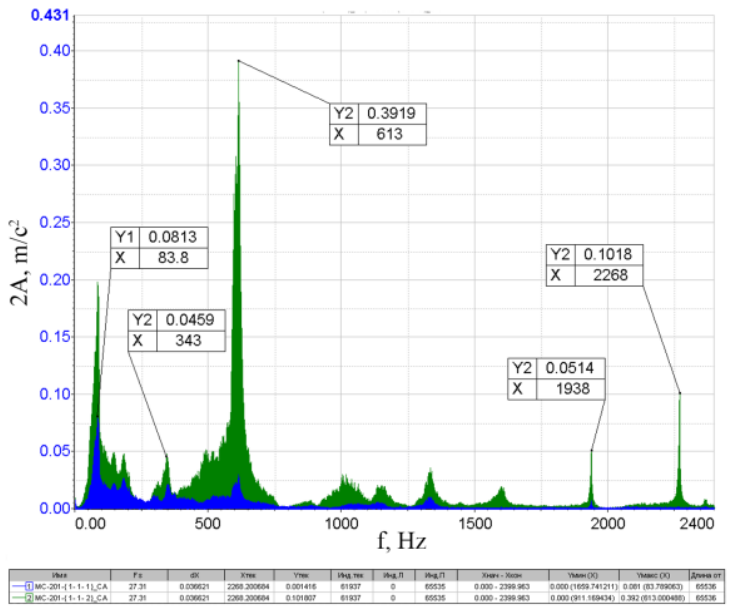

Fig. 8. First natural frequencies of guide vane (blue color - the sensor is centered; green color - the sensor is located at the edge). 


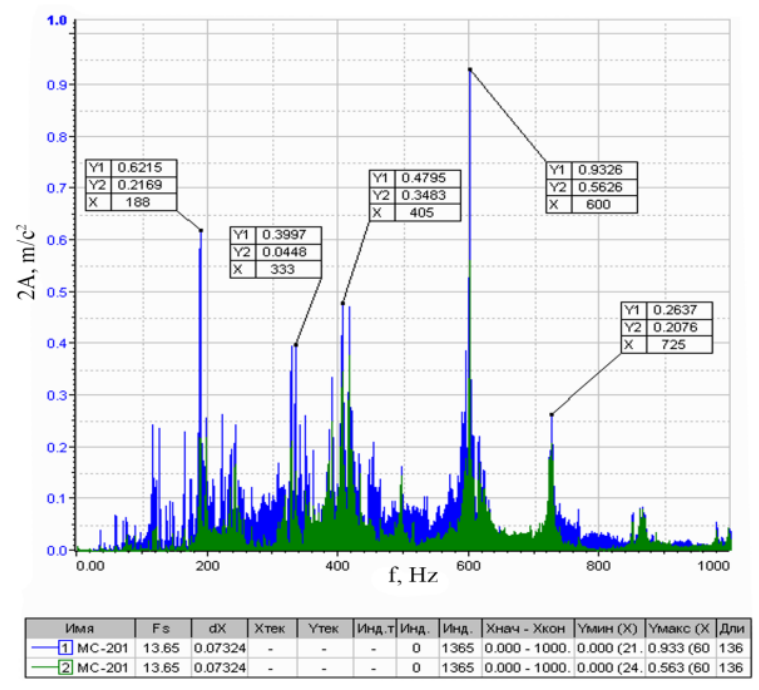

Fig. 9. First natural frequencies of runner blade (blue color - the sensor is located at outlet edge near band, green color - outlet edge near crown).

It can be seen from the figures that it's possible to determine the natural frequencies of the stay vanes and guide vanes with a certain accuracy. It is difficult to distinguish the natural frequencies of runner blade from a dense spectrum probably due to complex support and shape of blade.

The natural frequencies of the same elements in water will be $60-70 \%$ less than in air [1], [2]. In table 2 natural frequencies closed to main sound frequency $(300-370 \mathrm{~Hz})$ are shown.

Table 2. First natural frequencies of turbine water path elements.

\begin{tabular}{|c|c|c|c|}
\hline & Stay vane & Guide vane & $\begin{array}{c}\text { Runner } \\
\text { blade }\end{array}$ \\
\hline Natural frequency in air (measured), Hz & 286 & 613 & 600 \\
\hline $\begin{array}{c}\text { Natural frequency in water (calculated by division } \\
\text { on coefficient } 0,6 \ldots 0,7), \mathrm{Hz}\end{array}$ & $172-200$ & $368-429$ & $360-420$ \\
\hline
\end{tabular}

The calculated flow velocity for the optimum in the region of the outlet edge of guide vanes is in the $6-12 \mathrm{~m} / \mathrm{s}$ range. With thickness of outlet edge $10 \mathrm{~mm}$ frequency of Von Karman vortex shedding from guide vanes can be in the $120-240 \mathrm{~Hz}$ range and therefore much lower than the noise main frequencies.

Vortex shedding from runner blades may be closed to frequencies $300-370 \mathrm{~Hz}$ but the outlet edges of the runner blades and as well as guide vanes were modified by one-side cut $[3]$.

\section{Air injection to the turbine water path}

Air injection is a well-known method for reducing not only increased vibration [4] but also increased noise. Several methods of air injection to the turbine water path were also tested (figure 10). 
First way was to inject air through two pipes for depress water in synchronous condensing. During the tests, compressed air velocity on one of the two pipes (DN 150) was measured by air flow meter. The second way was to inject air from industrial air piping (with pressure $6 \mathrm{kgf} / \mathrm{cm}^{2}$ ) through piezometric pipes (DN 20) for measuring pressure in spiral case and in the area of wicket gate (figure 11).

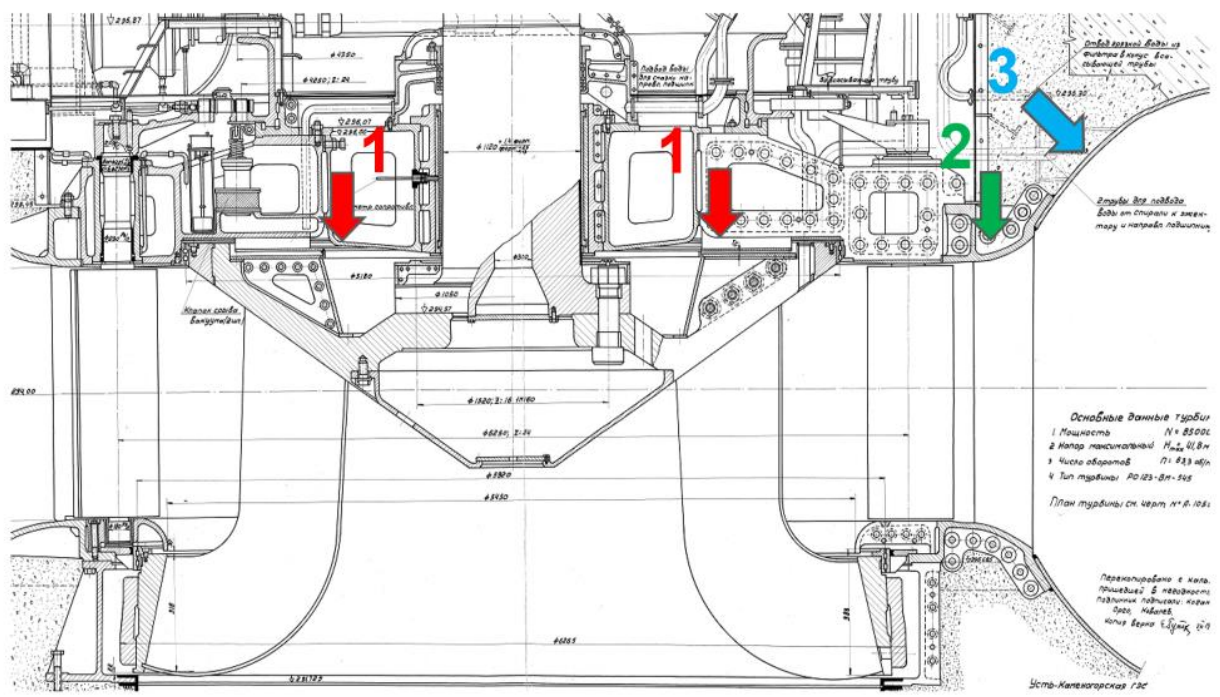

Fig. 10. General scheme of compressed air injection.

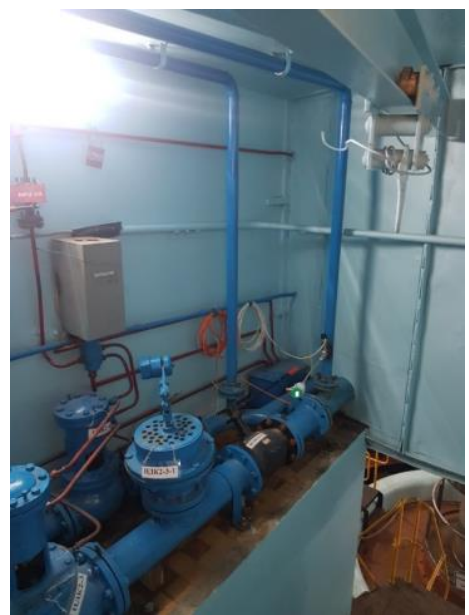

(1)

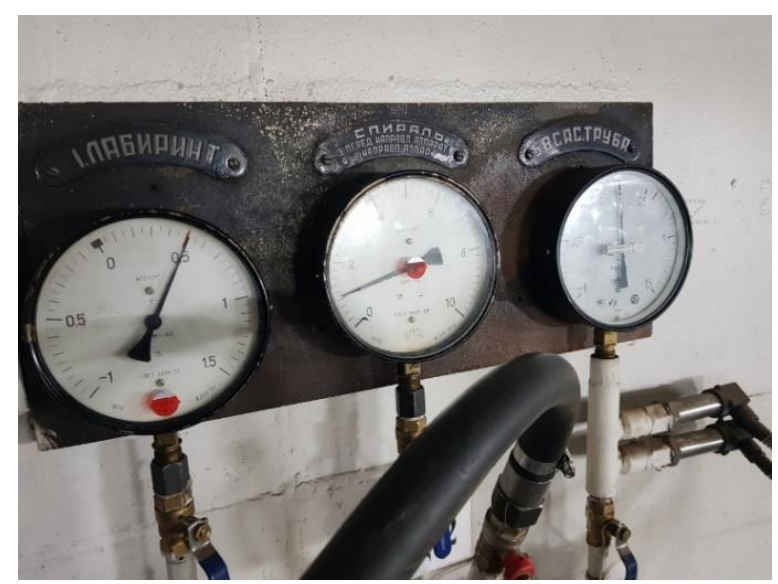

(2), (3)

Fig. 11. Compressed air injection ( 1 - two pipes for water depress in synchronous condensing; $2-$ piezometric pipe for measuring pressure in the area of wicket gate; 3 -piezometric pipe for measuring pressure in spiral case).

Two regimes ( $75 \mathrm{MW}$ and $85 \mathrm{MW}$ ) were selected in which air was injected by several methods. When air was injected through the pipes for water depress in synchronous condensing, the air velocity in one of the two pipes DN 150 was $\approx 92 \mathrm{~m} / \mathrm{s}$. Thus, the total air flow through two pipes was $\approx 3.3 \mathrm{~m}^{3} / \mathrm{s}$.

When air was injected through a piezometric pipe for measuring pressure, the air velocity can be estimated using the Torricelli formula: 


$$
V=\left(\frac{2 g \Delta p}{\rho}\right)^{\frac{1}{2}}
$$

where $\Delta p$ is the difference in air pressure at the beginning and at the end of the pipeline, $\rho$ is compressed air density. Thus, the estimated air flow through the pipe with a diameter of 20 $\mathrm{mm}$ and pressure at the end of pipeline $5.3 \mathrm{kgf} / \mathrm{cm}^{2}$ will be $\approx 0.04 \mathrm{~m}^{3} / \mathrm{s}$ (an order of magnitude less than in the first case).
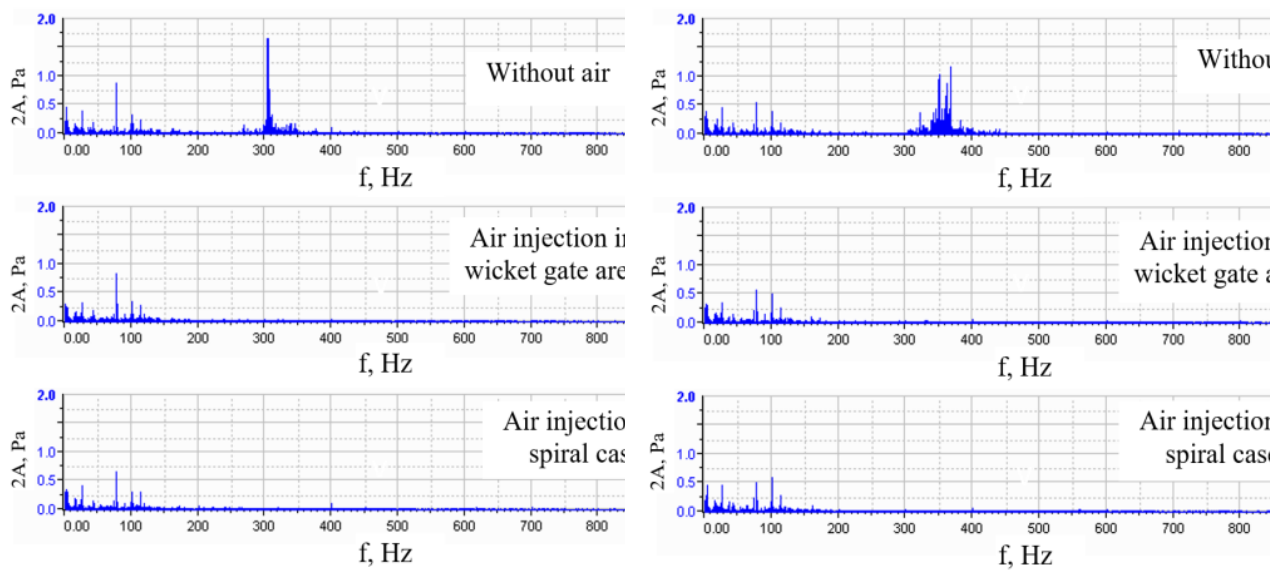

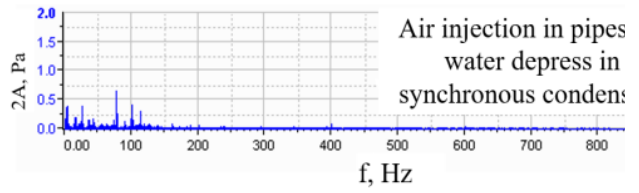

(a)

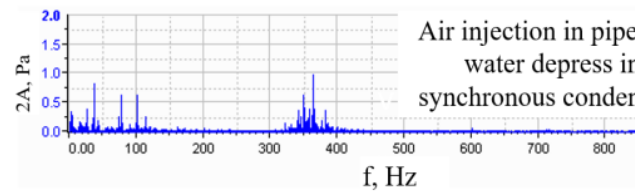

(b)

Fig. 12. Spectrum of sound pressure in turbine pit without air and with air injection at $75 \mathrm{MW}$ (a), 85 MW (b).

Acoustic noise with frequencies $\mathrm{f} \approx 300-310 \mathrm{~Hz}$ at $75 \mathrm{MW}$ disappeared with any method of air injection (figure 12, a).

Acoustic noise with frequencies $\mathrm{f} \approx 340-370 \mathrm{~Hz}$ at $85 \mathrm{MW}$ disappeared only when air is injected into the spiral case and into the area of the wicket gate (figure 12, b).

Similarly, the air injection also affects to the vibration of guide vanes: at $75 \mathrm{MW}$ vibrations with frequencies of $300-310 \mathrm{~Hz}$ disappeared with any method of air injection (figure 13, a). At $85 \mathrm{MW}$ vibrations with frequencies of $340-370 \mathrm{~Hz}$ disappeared only when air is injected into the spiral case and into the area of the wicket gate (figure 13, b). 

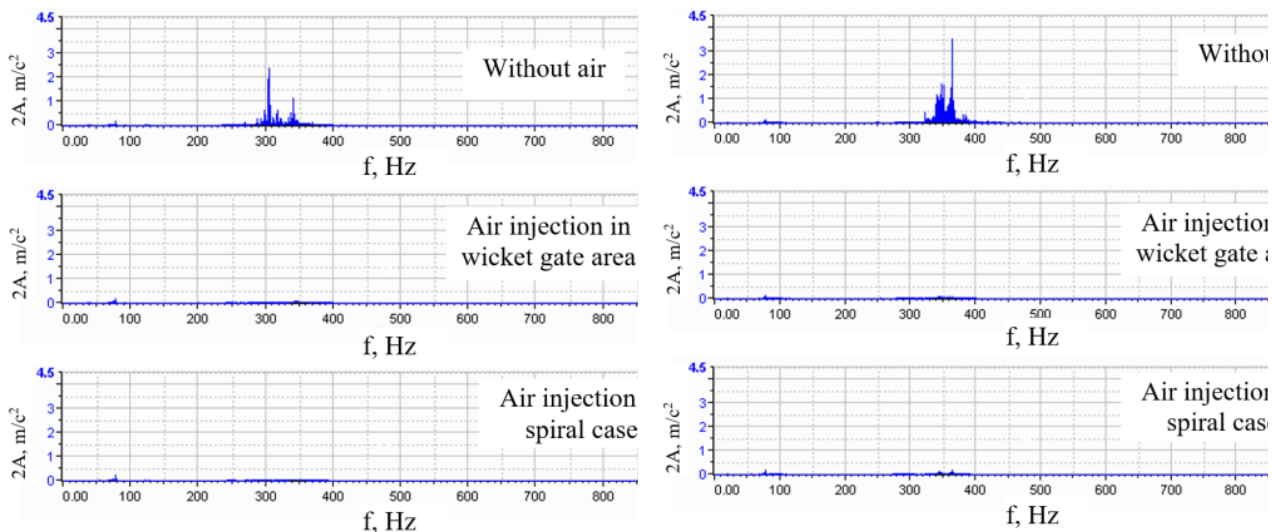

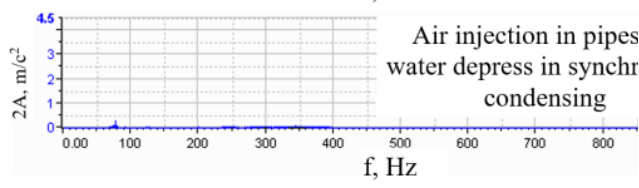

(a)

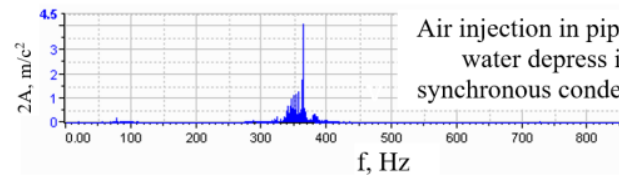

(b)

Fig.13. Spectrum of guide vane vibration in circumferential direction without air and with air injection at $75 \mathrm{MW}(\mathrm{a}), 85 \mathrm{MW}(\mathrm{b})$.

Air injected through a piezometric pipe into spiral case or wicket gate area probably was able to reduce or even destroy the structure of vortex (still water) starting from guide vanes.

Air injected through pipes for water depress in synchronous condensing was able to permeate through upper labyrinth into area between wicket gate and runner at $75 \mathrm{MW}$. Due to high pressure in front of the runner at $85 \mathrm{MW}$ almost all injected air escaped through discharge openings and didn't affect to the vortex.

\section{Conclusions}

Analysis of the Von Karman vortices showed that frequency of vortex shedding from guide vanes can be closed to frequencies $300-370 \mathrm{~Hz}$ but the outlet edges of the runner blades and guide vanes were modified by one-side cut.

Natural frequencies of hydro unit elements were determined by impact method. It is difficult to determine exactly the natural frequencies of elements in water due to approximate value of added mass of water, complex support and shape of some elements.

Perhaps the root cause noise may be vortices starting from the outlet edges of guide vanes that acting on the new runner blade system.

It is shown that the most effective method (including by the amount of air) to reduce acoustic phenomena was the air injection from industrial air piping into the spiral case and into the area of guide vanes. This solution has already been implemented on two hydro units with new runners. It is necessary to continue the research of the detected acoustic effect to determine the exact causes of it.

\section{References}

1. C. G. Rodriguez, Egusquiza E, Escaler X, Liang Q. W. and F. Avellan, Experimental investigation of added mass effects on a Francis turbine runner in still water Journal of Fluids and Structures 22, 2006, 699-712 
2. X. Huang and Escaler X., Added Mass Effects on a Francis Turbine Runner with Attached Blade Cavitation Fluids 4(2) 107, 2019

3. P. Doefler, Sick M. and Coutu A. Flow Induced Pulsation and Vibration in Hydroelectric Machinery (London: Springer) chapter4, 2012, 111-127

4. S. Fellenberg, Häussler W. and Michler W., High-Pressure Air Injection on a LowHead Francis Turbine 27th IAHR Symposium on Hydraulic Machinery and Systems IOP Conf. Series: Earth Environ. Science 22, 2014. 